\title{
The fallacy of a "task-negative" network
}

\section{R. Nathan Spreng*}

Laboratory of Brain and Cognition, Department of Human Development, Cornell University, Ithaca, NY, USA

${ }^{*}$ Correspondence: nathan.spreng@gmail.com
In a neuroimaging study of goal-directed cognition, brain activity will be significantly greater than baseline in a frontoparietal "task-positive" network (TPN). Further, a number of regions will be deactivated in the cinguloparietal "task-negative" network (TNN), or default network. Although this generic statement characterizes many findings in cognitive neuroscience, these network labels are imprecise at best, and also transmit a profound misconception about the functional role of the default network in cognition. The dichotomization of "taskpositive" and "task-negative" functional networks perpetuates the notion that the default network is not engaged in active cognitive processes. On the contrary, recent studies challenge this circumscribed view, demonstrating that: (1) the TPN comprises at least two functionally and anatomically distinct networks that play very different roles in cognition; and (2) the TNN (i.e., the default network) is not "task-negative" per se, but rather, is often engaged during goal-directed cognition, depending on the nature of the task. Further, (3) recent work demonstrates that components of these networks flexibly interact with one another based on task demands. These interactions raise important questions with respect to the role of the default network in goal-directed cognition and challenge the veracity and utility of a "TPN vs. TNN" distinction.

Engagement of the putative TPN is typically driven by tasks that confound demands for cognitive control and externally directed attention to visually presented stimuli. However, these processes, and their underlying functional neuroanatomy, can be dissociated. Cognitive control operations will engage an extended "frontoparietal control network," consisting of lateral prefrontal cortex (IPFC), precuneus, the anterior inferior parietal lobule, dorsal anterior cingulate cortex (dACC), and anterior insula (Vincent et al., 2008; Niendam et al., in press; Figure 1A, green regions). Visual attention will engage the "dorsal attention network," consisting of the frontal eye fields (FEF), inferior precentral sulcus, middle temporal motion complex $(\mathrm{MT}+)$, and superior parietal lobule (SPL; Figure 1A, red regions). While the constellation of regions across the frontoparietal control and dorsal attention networks (i.e., the TPN) may be co-active and coupled during task performance (cf. Badre et al., 2010; Grady et al., 2010; Gordon et al., in press), they need not be under all circumstances.

The default network was first identified by task-induced deactivations, or brain activity associated with a passive fixation "baseline" condition relative to specific attention-demanding visual tasks (Shulman et al., 1997; Raichle et al., 2001). Passive conditions, such as fixation, reliably engage the default network (Toro et al., 2008; Laird et al., 2009), which includes medial prefrontal cortex (MPFC), posterior cingulate cortex (PCC), superior and inferior frontal gyri, medial and lateral temporal lobes, and posterior inferior parietal lobule (pIPL; Buckner et al., 2008; Figure 1A, blue regions).

The terms "TPN" and "TNN" were first introduced by Fox et al. (2005). In this resting-state functional connectivity MRI study, spontaneous low-frequency BOLD signal fluctuations across the brain revealed an anti-phase, or "anti-correlated" relationship between two distinct and dissociable functional-anatomic networks (Fox et al., 2005) $)^{1}$. Their approach examined whole-brain correlations with a priori seed regions. TNN seeds were derived from an earlier meta-analysis of decreases in activity during tasks (i.e., relative to fixation;
Shulman et al., 1997) in MPFC, PCC, and pIPL; TPN seeds were derived from a study of cued attention and working memory and included IPS, FEF, and MT+, all within the dorsal attention network. The alignment of the TPN with the dorsal attention network persisted through the authors' initial papers (see Fox et al., 2005; Fox et al., 2006). Further, the default network was recognized by Fox et al. (2005) for its role in cognition, as evidenced in part by citing a mental inference study (Gusnard et al., 2001). Since the seminal observations of Fox et al. (2005), the TPN and TNN nomenclature has been widely adopted - likely due to its utility in explaining the persistence of dorsal attention network engagement and default network suppression across many tasks (Toro et al., 2008). However, the functional definition of the TPN, as described above, has expanded to encompass a broad spectrum of goal-directed attention tasks, encapsulating not only the dorsal attention network, but the frontoparietal control network as well ${ }^{2}$ (e.g., Grady et al., 2010; Hampson, 2010; Gordon et al., in press). In contrast, labeling the default network as "task-negative" has fortified its functional status as either deactivated by, uninvolved in, or even antithetical to, goal-directed cognition $^{3}$ (see Sonuga-Barke and Castellanos, 2007). Unfortunately, characterization of the default network as "task-negative" has inhibited scientific awareness of its critical functional role in active task conditions.

Conceptualization of the default network as a TNN began with the use of fixation as a passive baseline. As a base-

${ }^{1}$ The interpretability of negative correlations with the methodological use of mean signal regression has been questioned, as this approach introduces negative correlations between regions (Murphy et al., 2009). However, other observations without using mean signal regression demonstrate the same essential findings (Hampson et al., 2010; Chai et al., 2012).

${ }^{2}$ Drawing upon the work of Fox et al. (2005), the inclusion of elements of cognitive control to describe the TPN is exemplified by Hampson (2010). "Adopting the terminology of Fox et al. (2005), we refer to these cognitive control regions as 'task-positive' areas” (Hampson, 2010, p. 37).

${ }^{3}$ Also drawing upon the work of Fox et al. (2005), a literal interpretation of a TNN is applied by Sonuga-Barke and Castellanos (2007). “One such network, termed the 'default-mode' network is particularly observable during resting-states, and its activation is conjectured to be incompatible with goal-directed activity (Fox et al., 2005)" (Sonuga-Barke and Castellanos, 2007, p. 978). 

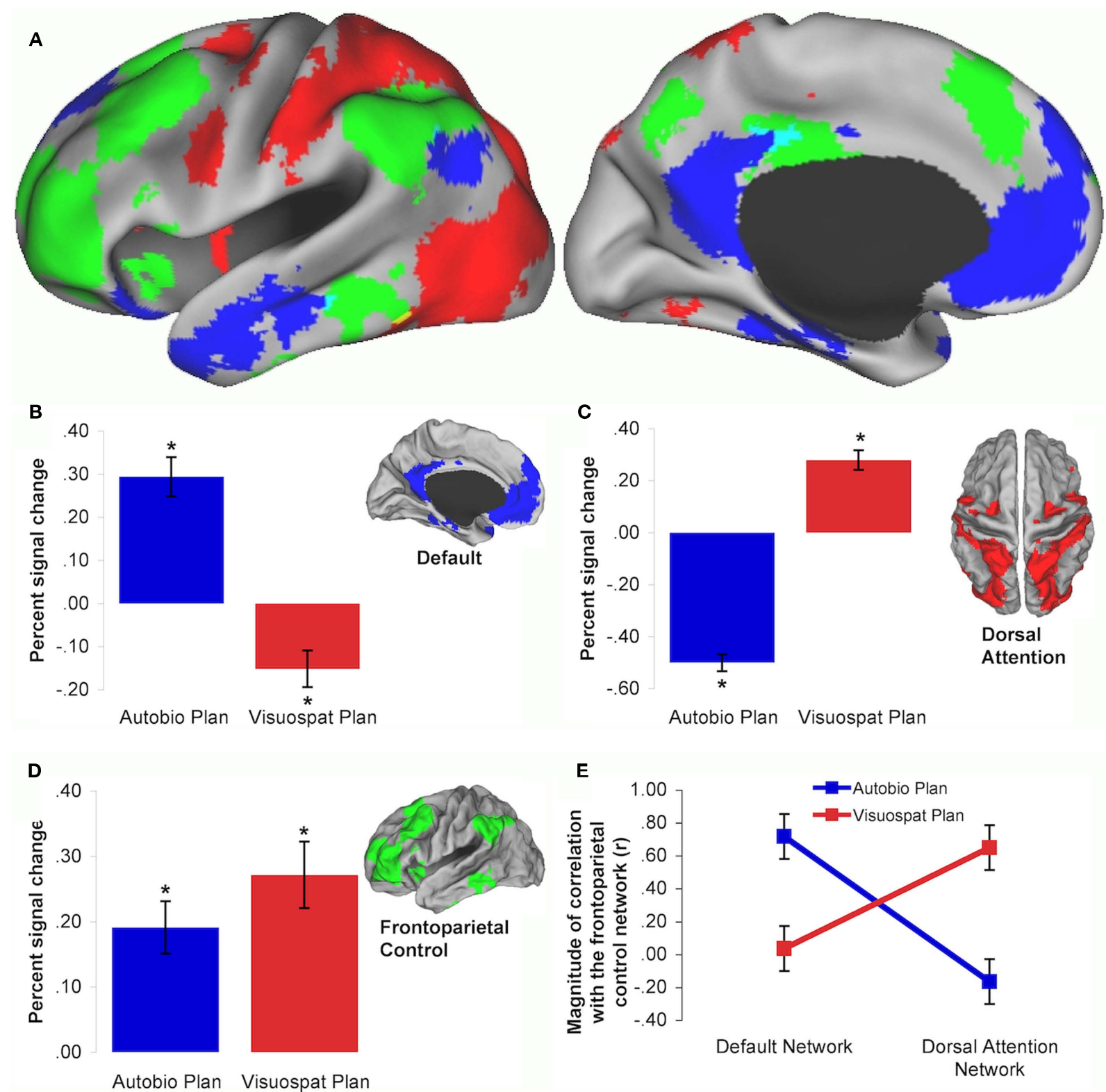

FIGURE 1 | (A) Intrinsic connectivity maps depicting the default (blue), dorsal attention (red), and frontoparietal control (green) networks of the brain. Mean and SEM of planning task-related percent BOLD signal change within each intrinsic connectivity network: (B) default network, (C) dorsal attention network, (D) frontoparietal control network. * indicates significant task difference in BOLD signal from baseline. (E) Frontoparietal control network coupling is

modulated by autobiographical and visuospatial planning tasks. Frontoparietal control network activity is coupled with the default network, and decoupled from the dorsal attention network, during autobiographical planning.

Frontoparietal control network activity is coupled with the dorsal attention network, and decoupled from the default network, during visuospatial planning. Data are reproduced from Spreng et al. (2010). line where no overt task is performed, fixation is a reliable method to localize the default network relative to active, externally directed, tasks. Far from being passive however, default activity during fixation is hypothesized to reflect unconstrained and internally focused cognitive processes (Buckner et al., 2008; AndrewsHanna, in press; see also Golland et al.,
2007; Golland et al., 2008). Moreover, default network activity is associated with many active cognitive processes that are internally focused, such as stimulusindependent thoughts or "mind-wandering” (McGuire et al., 1996; Mason et al., 2007; Christoff et al., 2009), self-reference (Gusnard et al., 2001; D’Argembeau et al., 2005) recollecting one's past or imagining one's personal future (Andreasen et al., 1995; Schacter et al., 2007; Spreng et al., 2009), scene construction (Hassabis and Maguire, 2007; Hassabis et al., 2007), social cognition (Iacoboni et al., 2004; Spreng et al., 2009; Spreng and Grady, 2010; Mar, 2011; Andrews-Hanna, in press), narrative comprehension (Mar, 2011), forming associations (Bar et al., 2007), and semantic 
memory (Binder et al., 2009). While many of these cognitive processes are stimulusindependent, spontaneous, and unconstrained, it is inaccurate to characterize them as "task-negative."

The value of the "TPN vs. TNN" nomenclature has been further eroded by observations that these two putatively opposed networks can be simultaneously engaged. Specifically, the default network may be co-active, and functionally coupled, with the frontoparietal control network under certain task conditions. This evidence emerges from studies of autobiographical planning, simulated problem-solving, evaluating one's creative work, mindwandering, social working memory, and scene construction (Spreng et al., 2010; Summerfield et al., 2010; Gerlach et al., 2011; Ellamil et al., 2012; Meyer et al., 2012). I recently investigated interactions between purported "task-positive" and "task-negative" brain regions using two planning paradigms: visuospatial planning, as assessed by the well-established Tower of London task, and autobiographical planning, as assessed by a novel task that required participants to devise personal plans in order to meet specific goals (Spreng et al., 2010). For example, "freedom from debt" constituted one of the goals in the autobiographical planning task. Participants viewed the goal and then saw two steps they could take toward achieving that goal ("good job" and "save money") as well as an obstacle they needed to overcome in order to achieve the goal ("have fun"). They were instructed to integrate the steps and obstacles into a cohesive personal plan that would allow them to achieve the goal. Autobiographical planning, similar to imagining personal future events (e.g., Addis et al., 2007), engaged the default network (Figure 1B). Visuospatial planning engaged the dorsal attention network (Figure 1C). Critically, both planning tasks engaged the frontoparietal control network (Figure 1D). Taskrelated functional connectivity analyses indicated that the frontoparietal control network dynamically coupled with the default network during autobiographical planning and with the dorsal attention network during visuospatial planning (Figure 1E). By demonstrating that the frontoparietal control network is actively engaged by two tasks that differentially rely upon either the default or dorsal attention network, we provided novel evidence that the frontoparietal control network may flexibly gain access to information processed in either domain (for a replication and additional data with older adults, see Spreng and Schacter, in press).

Other studies have also shown co-activation of components of these networks, with tasks simultaneously driving the default and frontoparietal control networks. Gerlach et al. (2011) examined brain activity during a problem-solving task involving mental simulations. They demonstrated that, relative to a semantic processing control task, problem-solving engaged several key regions within the default network, including MPFC and PCC, as well as a region of IPFC that has been linked with executive processing. These key default and frontoparietal control structures behaved as a functional network in a multivariate functional connectivity analysis. In a recent investigation of creative idea generation and evaluation, Ellamil et al. (2012) reported that generative tasks engaged default network regions. However, during an evaluative condition, default network regions coupled with regions of the frontoparietal control network, including IPFC. Further investigations by the same group (Christoff et al., 2009; Christoff, 2012) have demonstrated that the default network is co-active and coupled with regions of the frontoparietal control network during mind-wandering. Finally, a pair of studies demonstrated coactivation, where domain specific information modulated the default network and information load modulated the frontoparietal control network: one during a social working memory task (Meyer et al., 2012), the other involved integrating imagined objects into a scene (Summerfield et al., 2010).

Mirroring earlier evidence that functionally and anatomically dissociable dorsal attention and frontoparietal control networks interact as a functional network under specific task conditions (i.e., externally directed cognition), these more recent reports provide strong evidence for interactivity among default and frontoparietal control regions during internally directed cognition ${ }^{4}$. This emerging picture of dynamic interactivity among these three networks calls into question the orthodoxy of labeling functional brain networks as either TPN or TNN. These labels are more likely the byproduct of the desire for rigorously controlled experimental designs (i.e., externally directed stimuli) than meaningful descriptors of functional brain networks. However, research delineating and characterizing a taxonomy of neurocognitive networks is ongoing (e.g., Laird et al., 2011; Yeo et al., 2011). Indeed, the frontoparietal control network may be further fractionated into dissociable "salience" and "executive-control" networks (Seeley et al., 2007). Growing evidence for a complex and dynamically interacting network architecture highlight the importance of developing a stable nomenclature for functional brain networks. This will be an important consideration for future research.

Disregarding the false dichotomy of the TPN and TNN, competition between the dorsal attention and default networks may reflect competition between exogenous and endogenous loci of information processing. A critical function of cognitive control is to mediate this balance, by rapidly adapting thoughts and behaviors to changing internal states and evolving external environments. Cognitive control mechanisms promote mental flexibility by facilitating goal-directed actions and suppressing irrelevant ones. To achieve this, they must access and manipulate both exogenous and endogenous domains of information. In this way, the frontoparietal control network mediates internally and externally directed cognition by maintaining a dynamic balance between the default and attention networks (Vincent et al., 2008; Spreng et al., 2010, submitted; Smallwood et al., 2012; see also, Menon and Uddin, 2010).

\section{ACKNOWLEDGMENTS}

Thanks go to Gary Turner and Dale Stevens for helpful discussion and comments on earlier drafts of this manuscript, as well as to Dan Schacter for his support.

${ }^{4}$ Dynamic interactions between the default and frontoparietal control network have also been assessed using Granger causality during task performance (Gao and Lin, 2012) and examining low-frequency BOLD signal oscillations at rest (Deshpande et al., 2011). While compelling, these results may not be reliable (Smith et al., 2011). 


\section{REFERENCES}

Addis, D. R., Wong, A. T., and Schacter, D. L. (2007). Remembering the past and imagining the future: common and distinct neural substrates during event construction and elaboration. Neuropsychologia 45 , 1363-1377.

Andreasen, N. C., O'Leary, D. S., Cizadlo, T., Arndt, S., Rezai, K., Watkins, G. L., Ponto, L. L., and Hichwa, R. (1995). Remembering the past: two facets of episodic memory explored with positron emission tomography. Am. J. Psychiatry 152, 1576-1585.

Andrews-Hanna, J. R. (in press). The brain's default network and its adaptive role in internal mentation. Neuroscientist.

Badre, D., Kayser, A. S., and D'Esposito, M. (2010). Frontal cortex and the discovery of abstract action rules. Neuron 66, 315-326.

Bar, M., Aminoff, E., Mason, M., and Fenske, M. (2007). The units of thought. Hippocampus 17, 420-428.

Binder, J. R., Desai, R. H., Graves, W. W., and Conant, L. L. (2009). Where is the semantic system? A critical review and meta-analysis of 120 functional neuroimaging studies. Cereb. Cortex 19, 2767-2796.

Buckner, R. L., Andrews-Hanna, J. R., and Schacter, D. L. (2008). The brain's default network: anatomy, function, and relevance to disease. Ann. N. Y. Acad. Sci. $1124,1-38$.

Chai, X. J., Castanon, A. N., Ongur, D., and WhitfieldGabrieli, S. (2012). Anticorrelations in resting state networks without global signal regression. Neuroimage 59, 1420-1428.

Christoff, K. (2012). Undirect thought: neural determinants and correlats. Brain Res. 1428, 51-59.

Christoff, K., Gordon, A. M., Smallwood, J.,Smith, R., and Schooler, J. W. (2009). Experience sampling during fMRI reveals default network and executive system contributions to mind wandering. Proc. Natl. Acad. Sci. U.S.A. 106, 8719-8724.

D’Argembeau, A., Collette, F., Van Der Linden, M., Laureys, S., Del Fiore, G., Degueldre, C., Luxen, A., and Salmon, E. (2005). Self-referential reflective activity and its relationship with rest: a PET study. Neuroimage 25, 616-624.

Deshpande, G., Santhanam, P., and Hu, X. (2011). Instantaneous and causal connectivity in resting state brain networks derived from functional MRI data. Neuroimage 54, 1043-1052.

Ellamil, M., Dobson, C., Beeman, M., and Christoff, K. (2012). Evaluative and generative modes of thought during the creative process. Neuroimage 59, 1783-1794.

Fox, M. D., Corbetta, M., Snyder, A. Z., Vincent, J. L., and Raichle, M. E. (2006). Spontaneous neuronal activity distinguishes human dorsal and ventral attention systems. Proc. Natl. Acad. Sci. U.S.A. 103, 10046-10051.

Fox, M. D., Snyder, A. Z., Vincent, J. L., Corbetta, M., Van Essen, D. C., and Raichle, M. E. (2005). The human brain is intrinsically organized into dynamic, anticorrelated functional networks. Proc. Natl. Acad. Sci. U.S.A. 102, 9673-9678.

Gao, W., and Lin, W. (2012). Frontal parietal control network regulates the anti-correlated default and dorsal attention networks. Hum. Brain Mapp. 33, 192-202.

Gerlach, K.D., Spreng, R.N., Gilmore, A.W., and Schacter, D.L. (2011).Solving future problems: default network and executive activity associated with goal-directed mental simulations. Neuroimage 55, 1816-1824.

Golland, Y., Bentin, S., Gelbard, H., Benjamini, Y., Heller, R., Nir, Y., Hasson, U., and Malach, R. (2007). Extrinsic and intrinsic systems in the posterior cortex of the human brain revealed during natural sensory stimulation. Cereb. Cortex 17, 766-777.

Golland, Y., Golland, P., Bentin, S., and Malach, R. (2008). Data-driven clustering reveals a fundamental subdivision of the human cortex into two global systems. Neuropsychologia 46, 540-553.

Gordon, E. M., Stollstorff, M., Devaney, J.M., Bean, S., and Vaidya, C. J. (in press). Effect of dopamine transporter genotype on intrinsic functional connectivity depends on cognitive state. Cereb. Cortex.

Grady, C. L., Protzner, A. B., Kovacevic, N., Strother, S. C., Afshin-Pour, B., Wojtowicz, M., Anderson, J. A. Churchill,N., and Mcintosh,A.R. (2010).A multivariate analysis of age-related differences in default mode and task-positive networks across multiple cognitive domains. Cereb. Cortex 20, 1432-1447.

Gusnard, D. A., Akbudak, E., Shulman, G. L., and Raichle, M.E. (2001). Medial prefrontal cortex and self-referential mental activity: relation to a default mode of brain function. Proc. Natl. Acad. Sci. U.S.A. 98, 4259-4264.

Hampson, M. (2010). "Relating variations in network connectivity to cognitive function," in Analysis and Function of Large-Scale Brain Networks, ed. O. Sporns (Washington, DC: Society for Neuroscience), 35-42.

Hampson, M., Driesen, N., Roth, J. K., Gore, J. C., and Constable, R. T. (2010). Functional connectivity between task-positive and task-negative brain areas and its relation to working memory performance. Magn. Reson. Imaging 28, 1051-1057.

Hassabis, D., Kumaran, D., and Maguire, E. A. (2007). Using imagination to understand the neural basis of episodic memory. J. Neurosci. 27, 14365-14374.

Hassabis, D., and Maguire, E. A. (2007). Deconstructing episodic memory with construction. Trends Cogn. Sci. (Regul. Ed.) 11, 299-306.

Iacoboni, M., Lieberman, M. D., Knowlton, B. J., MolnarSzakacs, I., Moritz, M., Throop, C. J., and Fiske, A. P. (2004). Watching social interactions produces dorsomedial prefrontal and medial parietal BOLD fMRI signal increases compared to a resting baseline. Neuroimage 21, 1167-1173.

Laird, A. R., Eickhoff, S. B., Li, K., Robin, D. A., Glahn, D. C., and Fox, P. T. (2009). Investigating the functional heterogeneity of the default mode network using coordinate-based meta-analytic modeling. $J$. Neurosci. 29, 14496-14505.

Laird, A. R., Fox, P. M., Eickhoff, S. B., Turner, J. A., Ray, K. L., McKay, D. R., Glahn, D. C., Beckmann, C. F., Smith S. M., and Fox, P. T. (2011). Behavioral interpretations of intrinsic connectivity networks. J. Cogn. Neurosci. 23, 4022-4037.

Mar, R.A. (2011). The neural bases of social cognition and story comprehension. Annu. Rev. Psychol. 62, 103-134.

Mason, M. F., Norton, M. I., Van Horn, J. D., Wegner, D. M., Grafton, S. T., and Macrae, C. N. (2007) Wandering minds: the default network and stimulusindependent thought. Science 315, 393-395.

McGuire, P. K., Paulesu, E., Frackowiak, R. S. J., and Frith, C.D. (1996). Brain activity during stimulus independent thought. Neuroreport 7, 2095-2099.
Menon, V., and Uddin, L. Q. (2010). Saliency, switching, attention and control: a network model of insula function. Brain Struct. Funct. 214, 655-667.

Meyer, M.L., Spunt, R. P., Berkman, E. T., Taylor, S. E., and Lieberman, M.D. (2012). Evidence for social working memory from a parametric functional MRI study. Proc. Natl. Acad. Sci. U.S.A. 109, 1883-1888.

Murphy, K., Birn, R. M., Handwerker, D. A., Jones, T. B. and Bandettini,P.A. (2009). The impact of global signal regression on resting state correlations: are anti-correlated networks introduced? Neuroimage 44, 893-905.

Niendam, T.A., Laird, A. R., Ray, K. L., Dean, Y.M., Glahn, D. C., and Carter, C. S. (in press). Meta-analytic evidence for a superordinate cognitive control network subserving diverse executive functions. Cogn. Affect. Behav. Neurosci.

Raichle, M. E., Macleod, A. M., Snyder, A. Z., Powers, W. J., Gusnard, D. A., and Shulman, G. L. (2001). A default mode of brain function. Proc. Natl. Acad. Sci. U.S.A. 98, 676-682.

Schacter, D. L., Addis, D. R., and Buckner, R. L. (2007). Remembering the past to imagine the future: the prospective brain. Nat. Rev. Neurosci. 8, 657-661.

Seeley, W. W., Menon, V., Schatzberg, A. F., Keller, J., Glover, G. H., Kenna, H., Reiss, A. L., and Greicius, M. D. (2007). Dissociable intrinsic connectivity networks for salience processing and executive control. J. Neurosci. 27, 2349-2356.

Shulman, G. L., Fiez, J. A., Corbetta, M., Buckner, R. L., Miezin, F. M., Raichle, M. E., and Petersen, S. E. (1997). Common blood flow changes across visual tasks: II.Decreases in cerebral cortex. J. Cogn. Neurosci. 9, 648-663

Smallwood, J., Brown, K., Baird, B., and Schooler, J. W. (2012). Cooperation between the default mode network and the frontal-parietal network in the production of an internal train of thought. Brain Res. 1428, 60-70.

Smith, S. M., Miller, K. L., Salimi-Khorshidi, G., Webster, M., Beckmann, C. F., Nichols, T. E., Ramsey, J. D., and Woolrich, M.W. (2011). Network modelling methods for FMRI. Neuroimage 54, 875-891.

Sonuga-Barke, E. J., and Castellanos, F. X. (2007). Spontaneous attentional fluctuations in impaired states and pathological conditions: a neurobiological hypothesis. Neurosci. Biobehav. Rev. 31,977-986.

Spreng, R. N., and Grady, C. (2010). Patterns of brain activity supporting autobiographical memory, prospection and theory-of-mind and their relationship to the default mode network. J. Cogn. Neurosci. 22, 1112-1123.

Spreng, R. N., Mar, R. A., and Kim, A. S. (2009). The common neural basis of autobiographical memory, prospection, navigation, theory of mind, and the default mode: a quantitative meta-analysis. J. Cogn . Neurosci. 21, 489-510.

Spreng, R. N., and Schacter, D. L. (in press). Default network modulation and large-scale network interactivity in healthy young and old adults. Cereb. Cortex.

Spreng, R. N., Stevens, W. D., Chamberlain, J. P., Gilmore, A. W., and Schacter, D. L. (2010). Default network activity, coupled with the frontoparietal control network, supports goal-directed cognition. Neuroimage $53,303-317$.

Summerfield, J. J., Hassabis, D., and Maguire, E. A. (2010). Differential engagement of brain regions 
within a "core" network during scene construction. Neuropsychologia 48, 1501-1509.

Toro, R., Fox, P. T., and Paus, T. (2008). Functional coactivation map of the human brain. Cereb. Cortex 18, 2553-2559.

Vincent, J. L., Kahn, I., Snyder, A. Z., Raichle, M. E., and Buckner, R. L. (2008). Evidence for a frontoparietal control system revealed by intrinsic functional connectivity. J. Neurophysiol. 100, $3328-3342$.
Yeo, B. T., Krienen, F. M., Sepulcre, J., Sabuncu, M. R., Lashkari, D., Hollinshead, M., Roffman, J. L., Smoller, J. W., Zöllei, L., Polimeni, J. R., Fischl, B., Liu, H, and Buckner, R. L. (2011). The organization of the human cerebral cortex estimated by intrinsic functional connectivity. J. Neurophysiol. 106, 1125-1165.

Received: 23 February 2012; accepted: 23 April 2012; published online: 11 May 2012.
Citation: Spreng RN (2012) The fallacy of a "task-negative" network. Front. Psychology 3:145. doi: 10.3389/ fpsyg.2012.00145

This article was submitted to Frontiers in Cognition, a specialty of Frontiers in Psychology.

Copyright $\odot 2012$ Spreng. This is an open-access article distributed under the terms of the Creative Commons Attribution Non Commercial License, which permits noncommercial use, distribution, and reproduction in other forums, provided the original authors and source are credited. 